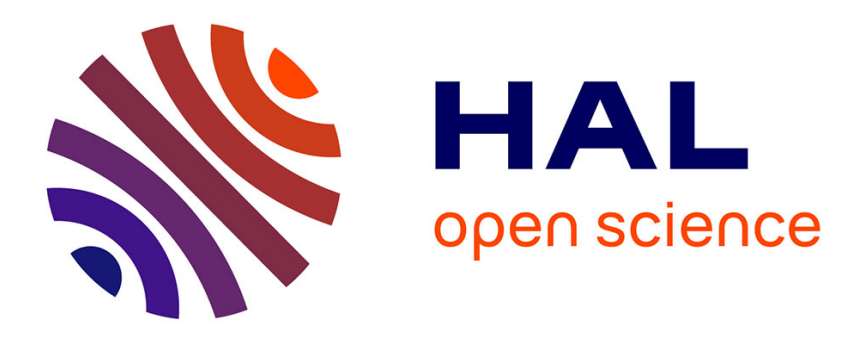

\title{
Generalization of the ideal crack model in eddy-current testing
}

\author{
Philippe Beltrame, Noël Burais
}

\section{To cite this version:}

Philippe Beltrame, Noël Burais. Generalization of the ideal crack model in eddy-current testing. IEEE Transactions on Magnetics, 2004, 40 (2 Part 2), pp.1366-1369. 10.1109/TMAG.2004.825433 . hal-00140338

\section{HAL Id: hal-00140338 \\ https://hal.science/hal-00140338}

Submitted on 18 Apr 2007

HAL is a multi-disciplinary open access archive for the deposit and dissemination of scientific research documents, whether they are published or not. The documents may come from teaching and research institutions in France or abroad, or from public or private research centers.
L'archive ouverte pluridisciplinaire HAL, est destinée au dépôt et à la diffusion de documents scientifiques de niveau recherche, publiés ou non, émanant des établissements d'enseignement et de recherche français ou étrangers, des laboratoires publics ou privés. 


\title{
Generalization of the Ideal Crack Model in Eddy-Current Testing
}

\author{
Ph. Beltrame and N. Burais
}

\begin{abstract}
In the ideal crack model in eddy-current testing frame, the field-flaw is equivalent to a current dipole layer on its surface. This model has shown its efficiency, as well for the computing accuracy, as for the CPU time. The goal of this paper is to improve this model taking into account the inclination, the conductance, and the low thickness of the crack.
\end{abstract}

Index Terms-Eddy-current testing, integral equations, modelization, thin crack.

\section{INTRODUCTION}

$\mathbf{T}$ HE THIN CRACK problem in eddy-current testing-i.e., the crack thickness $e$ (Fig. 1) is small compared with its other dimensions and to the skin depth $\delta$-constitutes a major difficulty for the simulation. It is commonly assumed that a surface crack is "ideal": being infinitesimally thin and allowing no current to flow across it. Then, Bowler [1] showed that the crack is equivalent to a current dipole surface of density $\mathbf{p}=$ $p \mathbf{n}$, where $\mathbf{n}$ is the crack normal (Fig. 1). A boundary integral method is used because only the flaw domain has to be meshed. The density is solution of an integral equation on the crack surface. The variation of the coil impedance is directly obtained from this density. Because of the hypersingular kernel of this equation, a regularization method is required to solve it [2]. This is applied to compute the density for a straight crack, i.e., $\alpha=0$ (Fig. 1), using a collocation method with second-order shape functions. The case of two straight parallel cracks in a tested piece was treated in [3]. A particular interest was related to the representation of the eddy-current distribution in the cracks neighborhood which posed a problem of evaluation of quasisingular integrals. This paper aims at generalizing the ideal crack model to take into account others parameters: equivalent crack conductance $\gamma$, inclination $\alpha$ and low thickness $e$. The originality of this last one is to avoid a three-dimensional (3-D) mesh for the crack by using the fact that the thickness is very thin.

Let us recall the relation between the perturbed electric current density $\mathbf{J}$, the unperturbed one $\mathbf{J}_{\mathbf{0}}$ and the density $p$ obtained by [1]

$$
\mathbf{J}\left(\mathbf{r}^{\prime}\right)-\mathbf{J}_{\mathbf{0}}\left(\mathbf{r}^{\prime}\right)=k^{2} \int_{S} \overline{\overline{\mathbf{G}}}\left(\mathbf{r}^{\prime}, \mathbf{r}\right) p(\mathbf{r}) \mathbf{n}(\mathbf{r}) \mathrm{d} \mathbf{S}(\mathbf{r})
$$

where $k$ is the propagation constant of the tested-piece, $\overline{\overline{\mathbf{G}}}$ is the electric-electric Green tensor and $\mathbf{r}^{\prime}$ a point in the tested piece.

Manuscript received July 1, 2003.

$\mathrm{Ph}$. Beltrame was with the CEGELY, Ecole Centrale Lyon, F-69131 Ecully, France. He is now with the Department of Fluidmechanics, Cottbus University, Cottbus D-03046, Germany (e-mail: beltrame@tu-cottbus.de).

N. Burais is with the CEGELY, Ecole Centrale Lyon, F-69131 Ecully, France (Noel.Burais@eea.ec-lyon.fr).

Digital Object Identifier 10.1109/TMAG.2004.825433

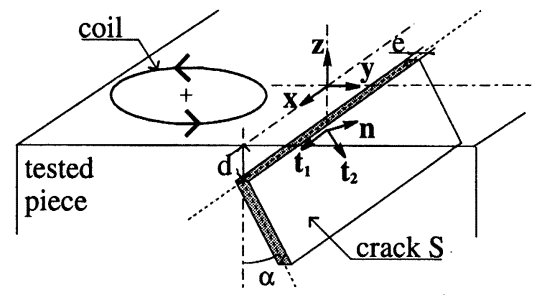

Fig. 1. Schematic configuration for the crack detection.

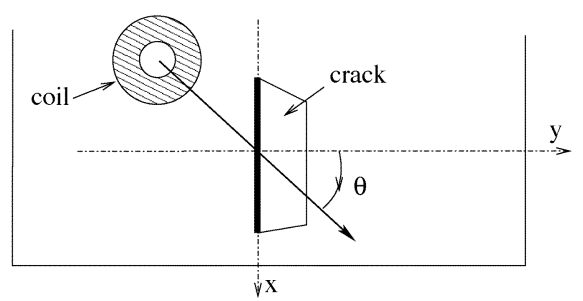

Fig. 2. Schematic representation of the coil displacement on the tested piece.

If it is on the crack then the integral has to be interpreted as the Finite Part of Hadamard, noted FP.

Finally the simulations are based on the parameters of the Team Workshop 15 [1], noted TW15 in the following, and we will indicate only the eventually changes. The sensor can move on the tested piece in the $\theta$ direction (Fig. 2).

\section{CRACK CONDUCTIVITY}

A relative conductivity of a crack was studied by Villone and Harfield [4] with two methods: finite element and integral equations. The formulation of the last one differs from our case and is applied for the high frequency approximation.

\section{A. Formulation}

The closure of crack may ultimately produce electrical contacts. In order to simulate the effects of current leakage across crack, an equivalent conductivity $\sigma_{f}$ of the crack is introduced. Then, the flow J.n crossing this surface is proportional to the density $p$ [4]. Then, from (1), we deduce

$$
\mathbf{J}_{\mathbf{0}} \cdot \mathbf{n}\left(\mathbf{r}^{\prime}\right)=\gamma \frac{p\left(r^{\prime}\right)}{\delta}-k^{2} F P \int_{S} \overline{\overline{\mathbf{G}}}\left(\mathbf{r}^{\prime}, \mathbf{r}\right) \cdot \mathbf{p}(\mathbf{r}) \mathrm{d} S(\mathbf{r})
$$

where the relative conductance is introduced

$$
\gamma=\frac{\delta \sigma_{f}}{e\left(\sigma_{0}-\sigma_{f}\right)}
$$

with $\sigma_{0}$ the conductivity of the tested piece.

With the collocation method described in [2], we have only to add the constant $\gamma$ in the contributions of the shape functions of the central node in the self-terms. 


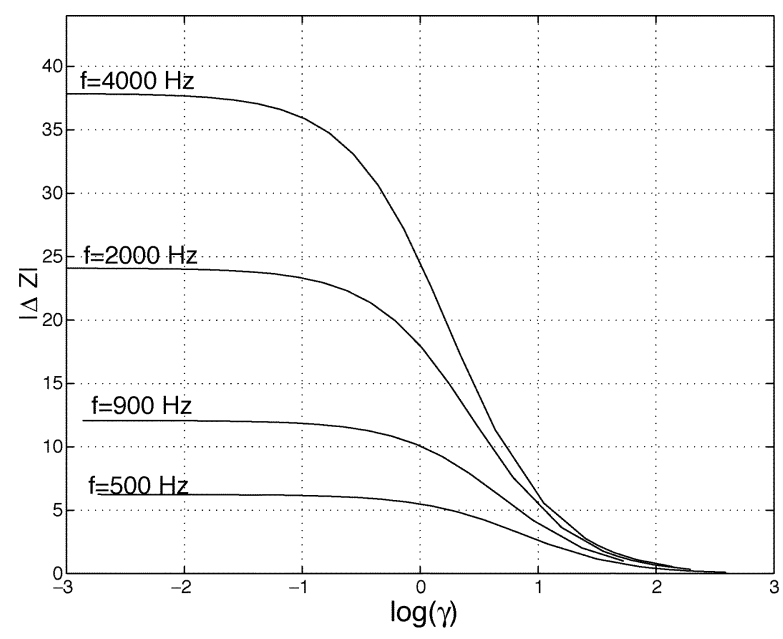

Fig. 3. Impedance change $|\Delta Z|$ vs the conductance $\gamma$.

\section{B. Results}

From the TW15, we have only changed the frequency and introduce a relative conductance of the crack. Fig. 3 shows the sensor response (impedance change) versus $\gamma$. Naturally the impedance change is decreasing while the conductance is increasing. For $\gamma<6.10^{-3}$ the difference between the impedance change with a nonzero conductance $\gamma$ and the ideal case is smaller than $1 \%$. More the frequency increases, more the sensor response falling gets sooner and faster: the response is $10 \%$ decreasing for $\gamma=0.2$ when $f=4000 \mathrm{~Hz}$ and for $\gamma=1.5$ when $f=500 \mathrm{~Hz}$. We have compared the variations of $\Delta Z$ between with or without conductance for a deplacement of the coil in the direction $\theta=90^{\circ}$ (Fig. 4). The shape of the variations of modulus or the dephasage are similar between both cases. We note that the dephasage is decreasing too if $\gamma \neq 0$

\section{INCLINED CRACK}

\section{A. Equations}

The equations deduced from (1) enable to compute the density $p$ and the impedance change $\Delta Z$ for all inclinations $\alpha$. However, we can notice that for the equation giving $p$, it is better to write the singular part of the integrand in the local basis of the crack (n, $\mathbf{t}_{\mathbf{1}}, \mathbf{t}_{\mathbf{2}}$ ) (Fig. 1), so it has exactly the same regularization expression than the one presented in [2] for a straight crack ( $\alpha=0$ ). Indeed, the singular kernel of the Green tensor is the one of an infinite piece in all directions and so it does not depend on the crack position.

The difficulty is for the eddy-current $\mathbf{J}$ representation at the crack surface. If the crack is straight, then eddy-current on the crack is the gradient of the density $p$. On the other hand, if the crack is inclined of an angle $\alpha$, it is necessary to take into account the reflexion terms $\overline{\mathbf{G}}_{R}$ [5]

$$
J_{t_{i}}^{\epsilon}\left(\mathbf{r}_{\mathbf{0}}\right)=J_{0 t_{i}}\left(\mathbf{r}_{\mathbf{0}}\right)-\frac{\epsilon}{2} \partial_{t_{i}} p+\int_{S} \mathbf{t}_{\mathbf{i}} \cdot \overline{\overline{\mathbf{G}}}_{R}\left(\mathbf{r}_{\mathbf{0}}, \mathbf{r}\right) \cdot \mathbf{p}(\mathbf{r}) \mathrm{d} S(\mathbf{r})
$$

where $\mathbf{r}_{\mathbf{0}}$ is a point of the crack surface, the index $t_{i}$ designates projection with respect the direction $\mathbf{t}_{\mathbf{i}}, \partial_{t_{i}}$ in the differentiation in this last direction, and $\epsilon= \pm 1$ is the positive or negative face of the crack.

\section{B. Results}

For the simulations, the crack has the inclination $\alpha$ and at the depth $d=0$ or $d=\delta$. We have chosen three different directions for the displacement of the coil $\theta=0^{\circ}, 45^{\circ}, 90^{\circ}$. The $\Delta Z$ amplitude decreases when the crack inclination $\alpha$ increases (Fig. 5). This relative decrease is more stressed when $\theta$ is increasing in the range $\left[0^{\circ} \ldots 90^{\circ}\right]$. The impedance change modulus during a coil displacement is more important when the coil is located on the side of the crack inclination. The dephasage varies a lot when the coil center is in the neighborhood of the crack (the last signature curve). For $\theta=45^{\circ}$ and $90^{\circ}$, then the $\Delta Z$ modulus decreases suddenly, whereas for $\theta=0^{\circ}$ the maximum is reached when the center coil is at the origin. Then, this last direction is the least efficient. The most efficient direction is obtained for $\theta=90^{\circ}$.

\section{THIN THICKNESS MODEL}

\section{A. Motivation}

The previous computing in the ideal crack model frame with second-order elements [2] and improving with special elements [6] shows that the impedance change differs from the experiment from some percents. This small difference could be explained by the fact: the real crack has a nonzero thickness and the ideal crack model neglects the influence of this last one. The volume integral method (VIM) overcomes this problem [7], but the mesh is $3-\mathrm{D}$ and the unknown is a density current vector with three components, instead a scalar unknown on a two-dimensional mesh in the ideal crack case. So the VIM requires more memory and CPU time. In fact, this model does not use the hypothesis of a thin crack.

The main idea of the thin thickness model is to deduce the impedance change, $\Delta Z(e)$, of a crack with the thickness $e$ from the impedance change $\Delta Z_{0}$ of the ideal one, adding a correcting term in the case $e \ll 1$. More precisely, we search the first-order approximation of the function $\Delta Z(e)$

$$
\Delta Z(e)=\Delta Z_{0}+e . K
$$

where $K$ is a constant to determine.

Yet, let us recall the expression of the impedance change in the generic case [8]

$$
\Delta Z(e)=\int_{S} \mathbf{J}_{\mathbf{0}} \cdot \mathbf{n}[v] d S+j \omega \int_{V} \mathbf{J}_{\mathbf{0}} \cdot \mathbf{A} d \tau
$$

where $\mathbf{A}$ : is the magnetic vector potential with Coulomb gauge and $v$ : the electric potential. This last one is discontinuous through the crack and the jump $[v]$ is related to the density $p$ density: $p=\left(\sigma_{0}-\sigma_{f}\right)[v]$. The first integral on the crack surface $S$ corresponds to the electric perturbation (deviation of the electric field lines) and the second one is the magnetic energy change in the crack volume $V$. Naturally, in the ideal crack model the crack volume tends to zero and this contribution vanishes. In the thin thickness model, this last one is taken into account. 


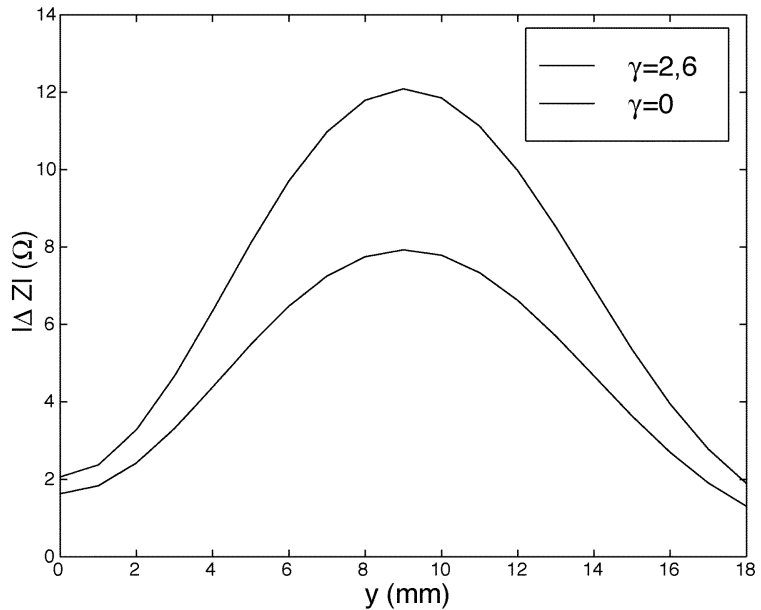

(a)

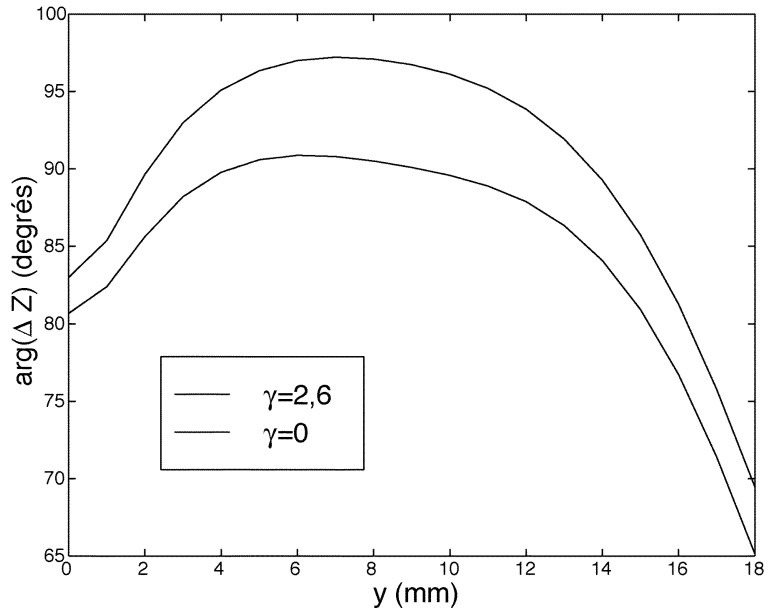

(b)

Fig. 4. Variations of the module (a) and dephasage (b) of the impedance change vs a sensor deplacement with or without a crack conductivity.

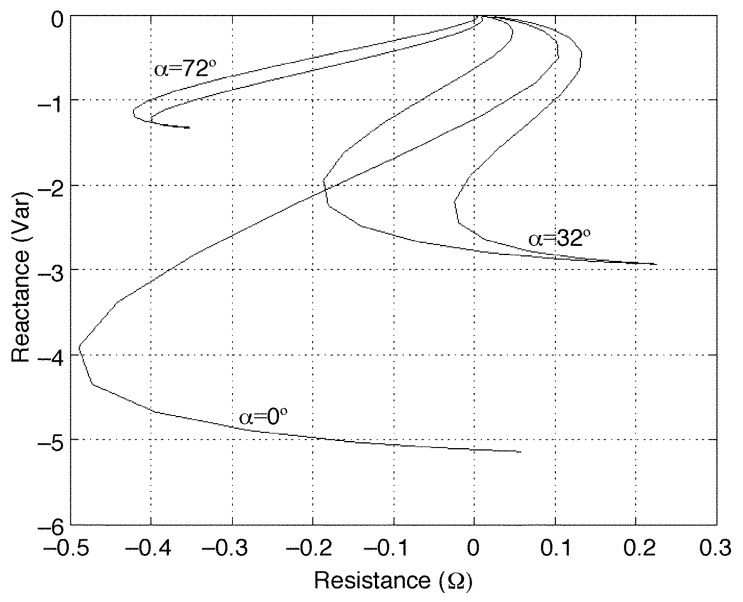

(a)

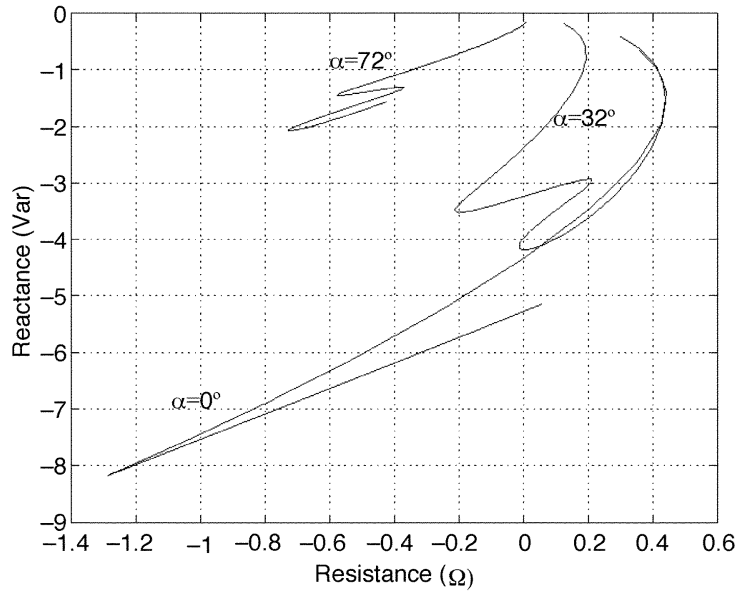

(c)

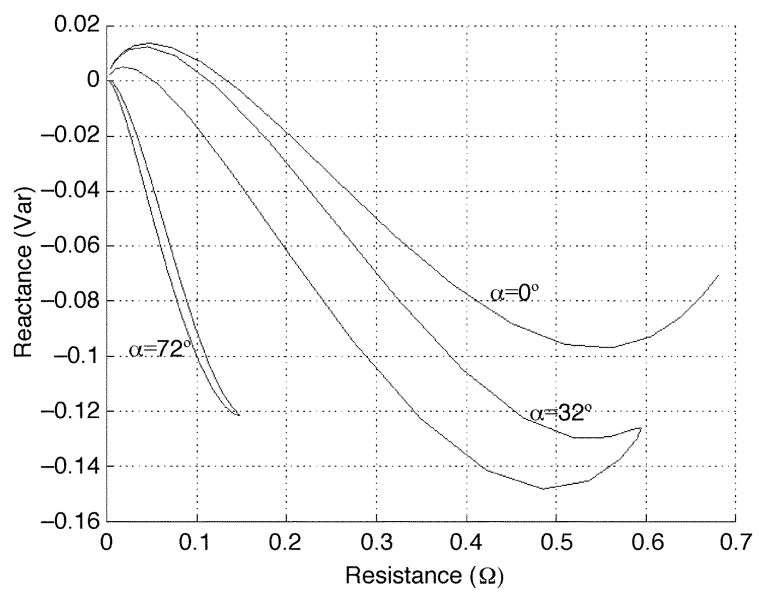

(b)

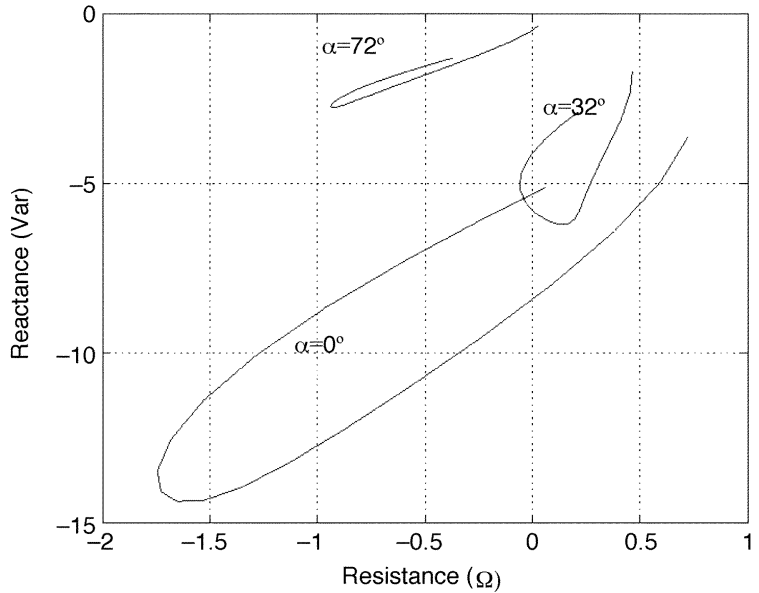

(d)

Fig. 5. Signatures for three different directions of coil displacements. (a) $\theta=0^{\circ}, d=0$; (b) $\theta=0^{\circ}, d=\delta$; (c) $\theta=45^{\circ}, d=0$; and (d) $\theta=90^{\circ}, d=0$.

Because of the variations of the potentials $\mathbf{A}$ and $V$ versus the thickness $e$ are small and the potential vector is continuous through the crack [5], we assume the following approximations:

1) the potential values of the potentials $\mathbf{A}$ and $V$ in the integrals are those computed in the ideal crack case;
2) the potential $\mathbf{A}$ is constant in the volume crack with respect to the normal $\mathbf{n}$.

Then, (6) is written as

$$
\Delta Z(e)=\Delta Z_{0}+e \int_{S} \mathbf{J}_{\mathbf{0}} \cdot \mathbf{A} d S=\Delta Z_{0}+e \cdot K .
$$




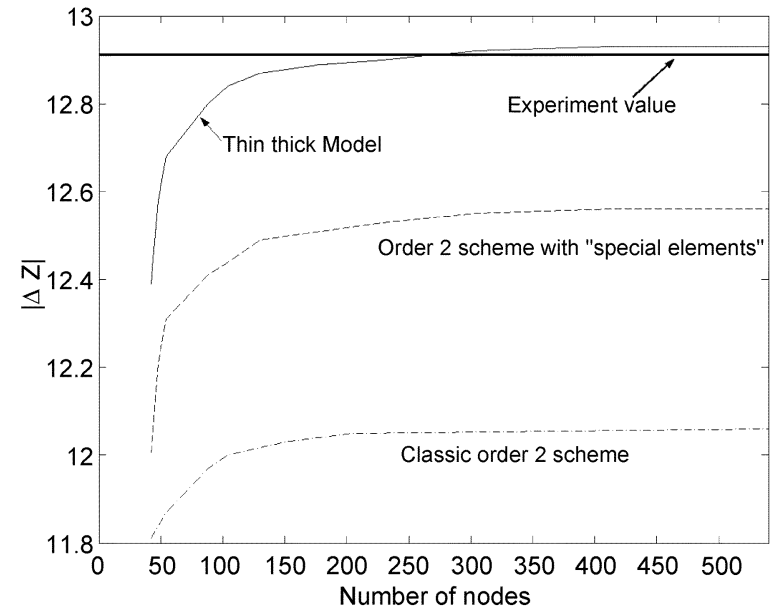

Fig. 6. Convergence with the thin thick model (solid line) and the ideal crack model (dashed lines) for the coil position $(x, y)=(9 \mathrm{~mm}, 0 \mathrm{~mm})$.

\section{B. Computing of the Constant $K$}

The main difficulty is to find the potential vector $\mathbf{A}$ at the crack surface $S$, in the Appendix we prove that the potential is given by

$$
\begin{aligned}
\mathbf{A . n}\left(\mathbf{r}_{\mathbf{0}}\right)= & -\gamma \frac{p\left(\mathbf{r}_{\mathbf{0}}\right)}{\delta}+F P \int_{S} p(\mathbf{r}) \frac{\partial^{2} G_{v}\left(\mathbf{r}, \mathbf{r}_{\mathbf{0}}\right)}{\partial n_{r} \partial n_{r_{0}}} d S_{r} \\
\mathbf{A . t} \mathbf{t}_{\mathbf{i}}\left(\mathbf{r}_{\mathbf{0}}\right)= & \left(\mathbf{E}_{\mathbf{0}}\left(\mathbf{r}_{\mathbf{0}}\right)+\int_{S} \overline{\overline{\mathbf{G}}}_{R}\left(\mathbf{r}, \mathbf{r}_{\mathbf{0}}\right)(p(\mathbf{r}) \mathbf{n}) d S_{r}\right) \cdot \mathbf{t}_{\mathbf{i}} \\
& -\int_{S} p(\mathbf{r}) \frac{\partial^{2} g_{R}}{\partial n \partial t_{i}}\left(\mathbf{r}, \mathbf{r}_{\mathbf{0}}\right) .
\end{aligned}
$$

The constant $K$ is obtained by the integration on the surface crack of the integrand $\mathbf{J}_{\mathbf{0}} . \mathbf{A}$. The integrand is evaluated at the nodes of the mesh and assuming that the integrand is quadratic on each element, the integration is easily deduced. Let us notice that the integrand cannot be evaluted at the crack edges because the regularization fails in this case (the gradient of $p$ is infinite).

\section{Validation}

In order to validate this model, the TW15 problem is solved. The computation of the ideal crack was improved by using special elements at the crack edges. When the thickness is taken into account, the theoretical value differs from less than $1 \%$ from the experiment while in the case of the ideal crack it was about $2.5 \%$ with special elements (Fig. 6). We have shown in [5] that the thickness influence depends strongly on the ratio $e / \delta$. So, the thin thickness model permits to perform the impedance change computing when $e / \delta<10 \%$

\section{CONCLUSION}

The ideal crack model was improved by introducing the formualtions for three parameters: $\gamma, \alpha$, and $e$. The study of the influence of these parameters allows determining their relevance in the inverse problem. The thin thickness model is relevant for $e / \delta<10 \%$ and the main advantage to a direct method, such the VIM, is that we have to add a correcting term after the pro- cessing. Furthermore, with these formulations, it will not be difficult to introduce a variable conductance and thickness.

\section{APPENDIX \\ EXPRESSION OF A ON THE CRACK SURFACE}

In the $(\mathbf{A}, V)$ formulation frame with the Coulomb gauge, the electric field on the crack surfaces $\epsilon= \pm 1$ is decomposed into two parts

$$
\mathbf{E}^{\epsilon}=j \omega \sigma_{0} \mathbf{A}^{\epsilon}-\nabla \mathbf{v}^{\epsilon}
$$

We can prove that the electric scalar potential $v$ is solution of the problem [5]

$$
\begin{aligned}
-\Delta v & =0 \text { in tested piece without crack } \\
\frac{\partial v}{\partial n} & =0 \text { on tested piece boundaries } \\
{[v] } & =\frac{p}{\sigma_{0}-\sigma_{f}} \text { on } S \\
{\left[\frac{\partial v}{\partial n}\right] } & =0 \text { on } S .
\end{aligned}
$$

The solution of this problem is well-known

$$
\nabla_{\mathbf{r}^{\prime} v}\left(\mathbf{r}^{\prime}\right)=\int_{S} \frac{p(\mathbf{r})}{\sigma-\sigma_{f}} \frac{\partial}{\partial n_{r}}\left(\nabla_{\mathbf{r}^{\prime}} G_{v}\left(\mathbf{r}, \mathbf{r}^{\prime}\right)\right) d S_{r}
$$

where $G_{v}$ is the elementary solution of the Poisson equation in the tested piece domain with Neumann boundaries. When $r^{\prime}$ tends to a point on $S$, then the last equation is interpreted as the finite part of Hadamard. Then for the component $\mathbf{n}$ we obtain the (8). For the tangential components, we obtain

$$
j \omega A_{t_{i}}=J_{t_{i}}^{\epsilon}-\epsilon F P \int_{S} p(\mathbf{r}) \frac{\partial^{2} G_{v}}{\partial n_{r} \partial t_{i r^{\prime}}} \mathrm{d} S(\mathbf{r}) .
$$

The dependence of the crack side $\epsilon$ is not real because the potential $\mathbf{A}$ is continuous through the crack. In fact, the singular part of the integral is $(\epsilon / 2) \partial_{t_{i}} p$, i.e., exactly the one of $\overline{\overline{\mathbf{G}}}$, because the strong singularity comes from the electrostatic part of Green functions [2]. Using (4), the discontinuities vanish and we obtain (9), where $g_{R}$ is the reflexion terms of the Green function $G_{v}$.

\section{REFERENCES}

[1] J. Bowler, "Eddy-current interaction with an ideal crack. The forward problem," J. Appl. Phys., vol. 75, no. 12, pp. 8128-8137, 1994.

[2] Ph. Beltrame and N. Burais, "Computing methods of hypersingular intergal applied to Eddy-Current Testing," IEEE Trans. Magn., vol. 38, pp. 1269-1272, Mar. 2002.

[3] - "Application of regularization method to compute eddy-current distribution near cracks," COMPEL, vol. 24, no. 4, 2002.

[4] F. Villone and N. Harfield, "Simulation of the effects of current leakage across thin cracks," in Electromagnetic Nondestructive Evaluation (IV). Amsterdam, The Netherlands: IOS Press, 2000, pp. 79-86.

[5] Ph. Beltrame, "Modélization de la Perturbation Électromagnétique au Voisinage D'une Fissure Mince. Application au Contrôle Non Destructif par Courants de Foucault," PhD thesis, Ecole Centrale, Lyon, France, 2002.

[6] - "Special elements at the crack edges in the eddy-current testing," presented at the ISEF Congr., Maribor, Slovenia, 2003.

[7] J. Bowler et al., "Eddy-current probe impedance due to a volumetric flaw," J. Appl. Phys., vol. 70, no. 3, pp. 1107-1114, 1991.

[8] Z. Badics et al., "An effective 3-D finite element scheme for computing electromagnetic field distortions due to defects in eddy-currents nondestructive evaluation," IEEE Trans. Magn., vol. 33, pp. 1012-1020, Mar. 1997. 